INNOVACIÓN

\title{
Metodología crítica para la investigación científica del derecho
}

\author{
Metodologia crítica para pesquisa científica do direito
}

Critical methodology for scientific research of law

\author{
José Ramón Narváez Hernández \\ Escuela Judicial Electoral y Universidad Nacional Autónoma de México
}

\begin{abstract}
RESUMEN La investigación jurídica se presume no científica. Muchos de los productores de investigación jurídica no consideran importante alcanzar los parámetros necesarios para poder interactuar con otras ciencias, ya que dicen que las peculiaridades del derecho lo harían una ciencia ad hoc. Lo cierto es que actualmente es necesario que la metodología jurídica sea más consciente de las necesidades sociales y los cambios generacionales para poder responder de mejor manera a los retos que se le presentan.
\end{abstract}

PALABRAS CLAVE Ciencia del derecho, metodología jurídica, enseñanza del derecho, investigación jurídica, posmodernidad y derecho.

ABSTRACT Legal research is presumed non-scientific. Many of the producers of legal research do not consider it important to reach the necessary parameters to be able to interact with other sciences because they say that the peculiarities of law would make it an ad hoc science. The truth is that today it is necessary that the legal methodology be more aware of social needs and generational changes in order to better respond to the challenges that arise.

KEYWORDS Law science, legal methodology, law education, legal research, postmodernity and law.

RESUMO A pesquisa jurídica é presumidamente não científica. Muitos dos produtores de pesquisas jurídicas não consideram importante atingir os parâmetros necessários para poder interagir com outras ciências, porque afirmam que as peculiaridades do direito o tornariam uma ciência ad hoc. A verdade é que hoje é necessário que a metodo- 


\begin{abstract}
logia jurídica esteja mais atenta às necessidades sociais e às mudanças geracionais, a fim de responder melhor aos desafios que surgem.
\end{abstract}

PALAVRAS-CHAVE Ciência do direito, metodologia jurídica, educação em direito, pesquisa jurídica, pós-modernidade e direito.

\title{
La noción itinerante de metodología
}

Decir que pertenecemos a una generación posmoderna podría resultar una justificación retóricamente válida, pero sociológicamente insostenible. La posmodernidad no nos debería llevar a concebirnos como una generación hermenéuticamente irresponsable.

Sumarse a una especie de relativismo moral no debería suponer el evitar establecer propuestas concretas para afrontar los problemas contemporáneos. Hablar de metodología supone, en principio, hacerse responsable de lo que se estudia y se propone, lo que llamaré «responsabilidad hermenéutica».

Actualmente, la llamada sociedad del conocimiento demanda más investigación, que debe hacerse bajo ciertos parámetros metodológicos, pero para lograrlo es necesario investigarlos previamente, así que esta especie de cinta de Moebius actualmente mueve un proceso que alimenta de manera vertiginosa el saber humano, a veces movido más por la inercia y la necesidad de innovación que por la previsión y la reflexión, ambas necesarias para generar la responsabilidad hermenéutica apuntada.

Bastaría citar a Ruiz a propósito de la importancia del tema en nuestros días:

Siendo el saber un elemento tan importante en la sociedad del conocimiento era obvio que la escuela suscitase gran interés entre los estudiosos de la formalización del conocimiento tácito y la innovación [...] Sin embargo, y a pesar de los estudios [...] la enseñanza es un ámbito que adolece de investigación y teorización de manera específica, en relación con la sociedad del conocimiento, y que las innovaciones dirigidas a ampliar el conocimiento en este ámbito carecen también de investigaciones concretas que codifiquen los conocimientos propios de la tarea o se dirijan a explicitar su dimensión tácita (2017:113).

La metodología propuesta ciertamente es crítica, escéptica si se quiere, pero laboriosa, ya que las incertezas implican una mayor responsabilidad. La búsqueda de respuestas se torna más intensa y más amplia. Nuestras pesquisas deben ser más integrales y más panorámicas. El pluralismo supone más trabajo, se trata de agotar en la medida de lo posible todas las potenciales respuestas a nuestro problema.

El fin del discurso supone la comprobación de más prácticas, la atención más profunda de la realidad, una vocación empírica más intensa. 
Consideraremos a la investigación como una actividad constante de búsqueda que de manera abductiva vamos construyendo sobre la base de una metodología itinerante, es decir, vamos trazando posibles rutas de análisis sobre los problemas.

La primera característica que encontramos con respecto a esta propuesta es la dinamicidad. Estamos acostumbrados a definir desde el inicio de toda investigación una metodología certera e invariable, pero después de ideas como el rizoma no podríamos pensar que existe un solo camino, que este es en línea recta y que deberíamos seguirlo indefectiblemente durante nuestra búsqueda:

Resumamos los caracteres principales de un rizoma: a diferencia de los árboles o de sus raíces, el rizoma conecta cualquier punto con otro punto cualquiera, cada uno de sus rasgos no remite necesariamente a rasgos de la misma naturaleza; el rizoma pone en juego regímenes de signos muy distintos e incluso estados de no-signos. El rizoma no se deja reducir ni a lo uno ni a lo múltiple (Deleuze y Guatari, 1994: 16).

La física cuántica demostró que el camino más corto entre un punto y otro no necesariamente es la recta. ¿Cómo podríamos concebir al método - que es un tipo de camino- como algo lineal aún? Estamos hablando de los planteamientos que la filosofía de la ciencia está esbozando a las ciencias sociales. Un rasgo de la posmodernidad es muy positivo, la complejidad es un factor que debemos asumir como parte de nuestras investigaciones. Esto supone la adquisición de nuevas competencias, aptitudes y actitudes.

Una metodología itinerante tendría como cometido principal la movilidad, la exploración de rutas y la generación de escenarios diversos en términos de la propuesta prospectivista.

De esta forma, el planteamiento del problema se convierte en una tarea fundamental. Imagino esta actividad inicial como la elaboración de un mapa mental o, mejor aún, un árbol de conocimiento con muchas ramificaciones, que también coincide mucho con el esquema rizomático:

Contrariamente al árbol, el rizoma no es objeto de reproducción: ni reproducción externa como el árbol-imagen, ni reproducción interna como la estructura-árbol. El rizoma es una antigenealogía, una memoria corta o antimemoria. El rizoma procede por variación, expansión, conquista, captura, inyección. Contrariamente al grafismo, al dibujo o a la fotografía, contrariamente a los calcos, el rizoma está relacionado con un mapa que debe ser producido, construido, siempre desmontable, conectable, alterable, modificable, con múltiples entradas y salidas, con sus líneas de fuga. Lo que hay que volver a colocar sobre los mapas son los calcos, y no a la inversa. Contrariamente a los sistemas centrados (incluso policentrados) de comunicación jerárquica y de uniones preestablecidas, el rizoma es un sistema acentrado, no jerárquico y no significante, sin general, sin memoria organizadora o autómata central, definido únicamente por una circulación de estados. Lo que está en juego en el rizoma es una 
relación con la sexualidad, pero también con el animal, con el vegetal, con el mundo, con la política, con el libro, con todo lo natural y lo artificial, muy distinta de la relación arborescente: todo tipo de «devenires» (Deleuze y Guatari, 1994: 17).

Una buena investigación - y esto aplica para casi todo tipo de investigación-comienza con buenas preguntas. Muchas personas desprecian en especial esta fase, ya que creemos por arrogancia que todas las preguntas son interesantes y/o relevantes. Además, se piensa que basta colocar signos de interrogación para hacer de cualquier tema una pregunta. Digamos que el establecimiento de metodologías adecuadas pasa en gran parte por el hecho de hacer buenas preguntas.

Las buenas preguntas evitan los siguientes riesgos: i) ceguera de taller, por la cual una materia se torna cerrada en tanto que la comunidad de intérpretes que la desarrolla genera un discurso y una gramática que resulta lógica para los que participan de dicha comunidad; y ii) currículo oculto, donde la presunta objetividad en la investigación científica y la tendencia a la acriticidad y el lenguaje políticamente correcto impide detectar las agendas políticas e ideológicas detrás de muchos proyectos de investigación.

Una metodología itinerante debe ser curiosa, proactiva, generosa, emancipadora, pero sobre todo innovadora. Esto supone mucha creatividad y un ánimo marcado de descubrimiento (Velásquez, 1994: 199). Más adelante hablaremos de las emociones, porque ciertamente la emoción que subyace debajo de un descubrimiento es un gran impulso para el investigador y una forma de didáctica para los estudiantes que se encuentran en el radio de acción.

La creatividad es modificable y puede ser aprendida, hay metodologías idóneas para incrementar la creatividad (Ford y Harris, 1992: 123), «el verdadero pensamiento científico es inherente al pensamiento creativo, pero no es inherente a todos los científicos» (Velásquez, 1994: 201). En definitiva, existen metodologías que incentivan la creatividad y las que no lo hacen. En la medida de lo posible, generemos las del primer tipo.

\section{¿Ciencia del derecho?}

Aún resuenan las incisivas expresiones de Kirschmann, quien en 1847 pronunció en una academia jurídica berlinesa -Juristische Gesellschaft zu Berlin - una extensa conferencia que tituló «Die Wertlosigkeit der Jurisprudenz als Wissenschaft» (La falta de valor de la jurisprudencia como ciencia), donde menciona que «tres palabras rectificadoras del legislador convierten bibliotecas enteras en basura» (1983: 29).

La queja era válida cuando se hacía a partir de una ciencia que buscaba problemas y soluciones dentro de un contexto meramente normativo. Los problemas normativos del ordenamiento son meros problemas de diseño. La recriminación no alcanza si el referente es la realidad social. Si el derecho es visto como un fenómeno empírico, entonces una reforma legislativa no es tan peligrosa. 
Entonces, la existencia de la ciencia del derecho depende de dos cosas: i) ¿Qué es el derecho?; y ii) ¿Qué es la ciencia?

El derecho es un concepto plurívoco, esto supone que existen diversas nociones sobre el mismo, pero deberíamos asumir la responsabilidad hermenéutica necesaria para elegir un objeto de estudio relacionado con el derecho menos cómodo que solo el normativo.

Vuelvo a la cuestión de hacer buenas preguntas. Podríamos simplemente preguntarnos qué norma sería necesaria para solucionar este problema o, mejor aún, cómo podemos solucionar este problema. Me parece que las acusaciones de Kirchmann van en ese sentido, se trata de una reflexión que nace sin pies. Las propuestas reformistas son simplemente un placebo.

Ahora abordemos la cuestión de lo científico. Existen también muchas nociones de lo que es la ciencia, pero si retomamos la responsabilidad hermenéutica, habría una que nos conecta de forma más eficaz con los problemas que aquejan a la sociedad y busca generar el mayor beneficio posible entre sus receptores.

Ciencia debería ser una labor de formulación compartida de preguntas acompañada por un trabajo de formulación de propuestas, las cuales deben ser sometidas a un proceso de falsación para descartar loogmatismos y ofrecer a la sociedad mejores respuestas posibles.

La ciencia jurídica ha sido relegada a una orilla, fuera de los grandes movimientos sociales, fuera del gran debate cultural. En gran medida la responsabilidad es de los mismos juristas que, herederos de algo más que un bimilenario y refinadísimo análisis científico, han pensado, discurrido, escrito en un lenguaje técnico de rara esotericidad, validísimo como comunicación interna entre expertos, pero instrumento aislante en el ámbito de la comunidad general a causa de su ya conocida total incomprensión por parte del ser humano común (Grossi, 1996: 46).

Ciencia es el nombre que se le da a un saber ordenado de forma que se considera valioso en un sentido por su estructura coherente y las expectativas que genera en torno a una serie de problemas, los cuales propone resolver.

La crítica posmoderna al cientificismo es la que insiste en que dicho saber no buscaba cumplir sus objetivos, sino generar un monopolio sobre el conocimiento a través de un control de las investigaciones y resultados, disciplinando las metodologías y censurando la crítica. Para ello, debía manejar un lenguaje críptico y una codificación compleja que servía para hacer inaccesible dicho conocimiento. La ciencia debería ser un asunto abierto y transparente, cercano a sus destinatarios.

El derecho no ha sido la excepción, pero aquí hay una carga más perversa en tanto que la ciencia jurídica justifica sistemas que podrían ser opresores o mermar los derechos y libertades. Por esto, el compromiso social del científico del derecho debería ser mayor y más explícito. 
La teoría del derecho como conocimiento tendiente a la organización de la vida civil y la solución de conflictos debe ser más cautelosa con respecto a sus bases científicas y, viceversa, la ciencia cautelosa de las bases teóricas. Por esto, una ética de la ciencia jurídica sería de gran ayuda.

\section{Metodología emocional}

Una metodología itinerante en nuestros días tendría en cuenta las emociones, incluso tomando en consideración la interesante crítica de Byung Chul-Han:

El capitalismo del consumo introduce emociones para estimular la compra y generar necesidades [...] En última instancia hoy no consumimos cosas, sino emociones. Las cosas no se pueden consumir infinitamente, las emociones en cambio sí. Las emociones se despliegan más allá del valor de uso. Así se abre un nuevo campo de consumo con carácter de infinito (2014: 72).

Lo que no podemos dejar de lado es la consideración de que las emociones son ahora tomadas en cuenta para la elaboración de proyectos y planes de diversa índole, incluidos, me parece, protocolos de investigación:

A pesar de la importancia de la dimensión afectiva para los procesos de aprendizaje y para la relación educativa propiamente dicha, los aspectos emocionales en educación continúan siendo, al día de hoy, un complejo desafío. Este desafío no es en modo alguno exclusivo de los contextos escolares, sino también de otros contextos, como los empresariales y de un buen número de entornos profesionales, que requieren del dominio de una amplia variabilidad de competencias emocionales para el logro eficaz de sus objetivos. Así pues, tanto si nos referimos a procesos educativos o a aquellos otros más específicos tales como los procesos de formación, o a todos aquellos que se apoyan o inciden en las relaciones humanas, en todos ellos, insisto, está involucrada la dimensión emocional (Núñez, 2006:66).

No se trata de sucumbir ante las emociones, sino ser conscientes de su peso. Como lo menciona el propio Han, en principio debemos distinguir entre afección, sentimiento y emoción, aunque quizá la idea de lo emocional englobe todo. Una reacción espontánea ante un evento genera algunas afecciones que, eventualmente, pueden llegar a constituir preconceptos. Pero existen emociones más arraigadas en nosotros que moldean nuestro sistema de pensamiento y que se enquistan a manera de prejuicios que a veces no somos capaces de explicitar. Una labor de objetividad puede ser muy ardua si existe la alexitimia (Sivak, Wiater y Lolas, 1997) suficiente que impida distinguir nuestros sentimientos con respecto a un objeto de estudio.

Por otro lado, hay un entrenamiento emocional que una vez que se ha elegido un tema, incluso de manera objetiva y desprejuiciada, nos genera una emoción mayor en relación con dar una respuesta que termine con una situación que consideramos 
deleznable o, por el contrario, incorporar acciones que consideramos necesarias. La emoción ayudaría a impulsar nuestro ánimo para emprender un trabajo investigativo de largo aliento, a solventar los retos y generar respuestas más profesionales en tanto que ponemos todo nuestro interés en el tema.

Sobre las emociones nos falta mucho por explorar en el campo de la enseñanza del derecho. Curiosamente, el camino que han seguido algunos filósofos del derecho norteamericano ha sido el de casos prácticos-literatura-estudio de las emociones (Nussbaum, 2008). Suena muy lógico porque ciertamente los casos prácticos comportan datos relevantes para el ejercicio del derecho, pero también implican muchas emociones de los sujetos involucrados en los mismos, de las víctimas, de los destinatarios de la norma judicial, del profesor, de los estudiantes, y sabemos muy poco cómo trabajar con todo esto.

\section{El círculo virtuoso de la investigación científica}

Ya he dicho más arriba que una buena investigación puede iniciar con una buena pregunta, pero ¿de dónde nacen las buenas preguntas? He denominado «círculo virtuoso» a un proceso que se da espontáneamente y que genera un flujo dialéctico que hace mover a toda investigación científica.

No es una relación lineal, pero sí simbiótica. Una buena pregunta te hace leer, una buena lectura te genera mejores preguntas y algunas respuestas. Cuando todo eso lo pones en blanco y negro, surge la necesidad de discutirlo. Una buena discusión en la cual se explicitan lecturas y textos redactados o por redactar genera buenas preguntas, y así sucesivamente.

Ahora desglosemos las actividades involucradas en este círculo:

Lectura: En los primeros años de formación escuchamos de nuestros maestros o compañeros citas que nos suscitan interés, lo que nos lleva a leer. A veces ese mínimo incentivo sirve para seguir buscando otras lecturas. A la postre, tenemos el hábito de la lectura, que normalmente nos lleva a discusión. Lecturas descriptivas o poco profundas llevan a planteamientos descriptivos y generales. Si bien la ley se lee, no puede considerarse una buena lectura, y lo mismo podríamos decir de las sentencias. La elección de lecturas es fundamental para realizar una investigación interesante e innovadora. Así, elegir lecturas nada multidisciplinarias generaría ceguera de taller, dogmatismo, alienación e impediría detectar el currículum oculto.

Redacción: Ciertamente, lo primero que uno piensa es que deberíamos mejorar la forma en que redactamos, incluida la ortografía y la gramática. Es claro que un mensaje mejor escrito es mejor interpretado. Aquí el consejo más sesudo tiene que ver con lo dicho en el punto anterior: leer ayuda a escribir mejor. Pero vayamos un poco más allá de las formas. Necesitamos contar mejores historias, debemos explorar otro tipo de narrativas. Deberíamos estar hartos de la crítica que nos hacen, de nuestro 
lenguaje hiperformalista, anacrónico, altisonante. Se ha insistido mucho en el asunto del lenguaje ciudadano y es cierto, deberíamos ser más democráticos al redactar, pero esto va más allá. Si estamos considerando que es necesario incorporar una metodología multidisciplinaria, entonces nuestra redacción debería ser coherente con ese fin. No escribimos solo para abogados o, peor aún, solo para quien se desenvuelve en un tribunal. Se trataría de un texto generoso también con el lenguaje, atractivo, estético. Por otro lado, debe haber una buena argumentación que prescinda de las falacias y el exceso de citas, que al final es otro tipo de narrativa falaz.

Comunicación: Los canales de comunicación son múltiples. La clase es quizá el principal recurso cuando se le utiliza honestamente. El profesor que ha repetido durante muchos años el mismo manual a través de la clase magistral difícilmente entablará diálogo con sus alumnos. En palabras de Elgueta y Palma:

En este caso se la entiende como expresión de una práctica docente caracterizada por el verbalismo o uso intensivo del discurso. Es sinónimo de lección catedrática. Se la relaciona con una enseñanza pasiva y se la contrapone a la clase activa. Se señala que los profesores actúan como conferencistas y los estudiantes como sujetos pasivos (2014: 908).

La clase debería ser uno de los espacios privilegiados para la comunicación de descubrimientos científicos, ya que el cerebro del estudiante de derecho es curioso en los primeros años y puede generar enfoques diversos a los resultados. Los seminarios de discusión, los foros y congresos son otras maneras de exponer el desarrollo de una investigación. Algunos son especialmente diseñados para investigaciones en ciernes, pero también los hay para investigaciones finalizadas con especialistas en los temas. Por último, no desdeñaría la plática coloquial con amigos, familiares y cualquier otra persona con la que podamos compartir impresiones. Mejor si son personas directamente involucradas con el problema.

\section{El perfil del abogado como rezago de la investigación científica}

Nada se ha escrito sobre el particular y, sin embargo, es un mal por todos conocido. La prepotencia y arrogancia del abogado resulta inconveniente para emprender un trabajo de investigación crítico. La profesión jurídica es altamente reaccionaria y conservadora. El abogado se piensa institucional y último baluarte del Estado. Por eso he insistido en el compromiso social, ya que debe impulsarse como un elemento fundamental para cualquier metodología. No coinciden las expectativas sociales y políticas actuales con la parsimonia del operador jurídico que se contagia a la academia y el salón de clases (Molina, 2009: 52).

Las investigaciones en el ámbito del derecho no se distinguen por su creatividad, ni mucho menos por su multidisciplinariedad. Hay algo en la enseñanza del derecho 
que nos obliga a actuar de manera acrítica. Los trabajos de investigación deben ser descriptivos, en su caso exegéticos; se glosa la ley y la jurisprudencia; se hacen sugerencias para la re-forma de las instituciones; se adosa con un poco de citas y un capítulo de antecedentes históricos.

El abogado además está diseñado para cumplir requisitos. En algunos casos incluso hay hasta un cierto fetichismo en las formas, formatos y formularios. Con la aparición de la investigación cuantitativa este fetichismo ha aumentado. Citar sentencias por su numeración como se hacía con los artículos de la ley es un hobby muy practicado. Entre los estudiantes de posgrado hay gran preocupación por acreditar, incluso cuando han emprendido un ambicioso trabajo de investigación. Ahora la investigación misma se presenta como una manera ambiciosa de titulación frente a los múltiples esquemas para obtener un título de manera simplificada. Un trabajo de investigación no puede ser solo el número de cuartillas, el número de citas -mayormente autores varones y extranjeros - o un compendio legal o jurisprudencial, debería ser una buena idea desarrollada y con una propuesta con impacto social.

Desgraciadamente, para el académico la cuestión no es distinta. Informes cada vez más sofisticados que piden publicaciones en determinados medios y el opresivo sistema de puntos hacen del investigador un autómata en búsqueda de resultados rápidos y eficaces. Las buenas tesis están desapareciendo porque no otorgan altos puntajes.

El abogado no se siente científico social o humanista, se considera asimismo como una especie de asesor del sistema. Las investigaciones tienen más el cariz de policy papers. De hecho, hay una creciente necesidad de justificar los trabajos de investigación en el ámbito jurídico a través de demostrar que serán traducidos en un documento de política pública. Lo anterior incluso serviría para obtener financiamiento a la investigación, y no está mal que una investigación pretenda ser financiada o terminar como política pública, pero pocos han advertido el riesgo de disciplinamiento de las investigaciones. Finalmente, en los comités que evalúan las investigaciones hay académicos que replican la ceguera de taller y consciente o inconscientemente involucran el currículo oculto. Termina muchas veces siendo la investigación científica un gran lobby o, en el mejor de los casos, una negociación estilo Harvard.

\section{Metodología crítica}

A lo largo del texto he apuntado algunos posibles elementos de lo que podría ser una metodología crítica. Como un apunte inicial, tomaremos los elementos que señala Wolkmer como enfoque crítico:

Las expresiones teoría jurídica crítica, crítica jurídica o pensamiento crítico en el derecho, usadas indistintamente [...] deberán ser entendidas como el profundo ejercicio reflexivo de cuestionar lo que está normativizado y oficialmente consagrado 
(en el plano del conocimiento, del discurso, de las instituciones y del procedimiento práctico normativo) en una determinada formación social, y como la posibilidad de concebir otras formas no alienantes, diferenciadas y pluralistas de práctica jurídica [...] no es otra cosa que la formulación teórico-práctica potencialmente capaz de buscar pedagógicamente otra dirección u otro referencial epistemológico que responda a las contradicciones de la presente modernidad (2017: 14).

Entonces, partimos de la otredad o alteridad, de una dialéctica simple, del modo dogmatizado en que se han hecho las cosas y los «otros» posibles modos de hacerlas. Por ejemplo, frente al hermetismo de algunas ciencias como es el caso del derecho, resulta evidente que la multidisciplinariedad tendría que figurar dentro de esa posible lista de elementos para la crítica, un concepto ampliamente citado pero que se entiende poco frente a otros de parecida índole como la intra o interdisciplinariedad, y más recientemente la transdisciplinariedad. En cualquiera de los casos se trata de un ejercicio de exteriorización del conocimiento, de equiparamientos, de cruces y comparaciones. Así, una institución, un tema, un problema y las soluciones pueden ser localizadas en otras áreas que en principio no teníamos identificadas, a veces dentro del mismo derecho (inter), a veces dentro de las ciencias afines (intra), a veces más allá (multi/trans).

Los más puristas apuntarán que el riesgo es la extrapolación, pero muy seguramente se resguardarán detrás del muro de comodidad que da la experticia de una sola materia. Entonces, tornaríamos sobre el rizoma que rehúye a la univocidad y la plurivocidad, o propondríamos una metodología indisciplinada y emancipadora:

La práctica de los pedagogos se sostiene en la oposición de la ciencia y la ignorancia. Los pedagogos se distinguen por los medios que eligen para volver sabio al ignorante: métodos duros y suaves, tradicionales o modernos, pasivos o activos, cuyo rendimiento puede ser comparado [...] pero en realidad no hay nada que comparar [...] no se trata de dos métodos, la comparación se da entre dos inteligencias y dos concepciones del orden intelectual [...] la vía rápida no era la de una pedagogía mejor, sino la de la libertad (Rancière, 2008: 29).

La mejor metodología es la que emancipa, la que libera, no la que supuestamente reduce la ignorancia. Se trata de una metodología adecuada encontrada a través de la curiosidad y con una alta carga de emoción por el descubrimiento.

Natalia Fernández nos propone un sencillo itinerario a partir de la lectura a Onfray:

Se entiende que la filosofía es una actividad reflexiva y crítica, por tanto, práctica sobre la realidad. En este sentido se plantea también la necesidad de que la actividad filosófica sea contextualizada. Asimismo, se parte de una visión agonista de la realidad según la cual la Historia de nuestra cultura occidental debe ser analizada 
como una lucha de opuestos. Por ello se plantean las siguientes oposiciones: i) lo oculto frente a lo manifiesto; ii) lo práctico frente a lo teórico; iii) lo crítico frente a lo dogmático; iv) la razón de los vencidos frente a la opresión de los vencedores; v) el cuerpo frente al espíritu (2014: 15).

Crítica entonces en el sentido más dialéctico, es decir, como la argumentación permanente sobre las propias afirmaciones. Como inquietud, como insaciabilidad epistémica, como curiosidad hermenéutica y como preocupación social.

Ahora bien, una metodología crítica con conciencia social y aplicada debería superar algunas de las siguientes cuestiones esbozadas por Nelly Richard:

Por un lado, se trataría de recorrer la distancia que separa a la crítica de una red ampliada de interlocuciones sociales que debe exceder la figura del destinatario trazado por las especializaciones académicas, para generar necesarias «zonas de contacto» entre discurso intelectual y materias públicas. Por otro lado, se trataría de preocuparse por la distancia desde la cual enunciar la posición crítica para que dicha posición no se encuentre ni demasiado cercana del objeto a criticar (caso en el cual el discurso de la crítica queda miméticamente cautivo de los reflejos más superficiales de la actualidad creada por los medios de masas) ni tampoco demasiado lejana de ese objeto (caso en el cual se separa demasiado de aquel horizonte mediano de comprensión social que busca afectar y transformar) [...] Por un lado, huir de la evanescencia y liviandad de la coyuntura y, por otro, estar lo suficientemente atentos para detectar los temas capitales de la sociedad (2000: 44).

La crítica latinoamericana además ha apuntado que un requerimiento necesario -incluso a nivel identitario- es la descolonización:

La filosofía intercultural crítica rechaza cualquier esencialismo o purismo cultural y sostiene que todas las culturas de este planeta son el resultado de un proceso complejo y largo de «inter-trans-culturación». Por lo tanto, el objetivo del proceso de «descolonización» no puede significar la vuelta al statu quo ante, ni a un ideal bucólico y romántico de culturas «no contaminadas» [...] «Colonialidad» no es el hecho («neutral») de que todas y todos somos producto de este proceso humano de la inter-trans-culturación - que es un hecho histórico-, sino que contiene un aspecto analítico y crítico que tiene que ver con involuntariedad, dominación, alienación y asimetría de estructuras políticas, injusticia social, exclusión cultural y marginación geopolítica. En los últimos años, un gran número de publicaciones de las ciencias sociales -especialmente en América Latina- dan testimonio de este nuevo enfoque de analizar la «colonialidad» existente y del proceso de «descolonización» en la era de la globalización neoliberal. En estos trabajos, se intenta hacer una lectura crítica de la «colonialidad» latinoamericana en torno a las tres categorías de raza (lo «étnico»), trabajo (lo «económico») y género (lo «social»), siempre bajo la hermenéutica de sospecha del eurocentrismo, capitalismo y androcentrismo vigentes en el proyecto actual de la globalización neoliberal (Estermann, 2014: 10). 
Es una metodología de la sospecha, que evidencia nuestros prejuicios y a través de ellos nuestras dependencias, nuestra falta de libertad. En alguna ocasión he sugerido un ejercicio llamado «acción afirmativa epistémica». Las acciones afirmativas tienen como principal objetivo reducir brechas de desigualdad a través de actos que logren equiparar a sujetos que, de otra manera, por las circunstancias estarían en desigualdad. Las acciones afirmativas epistémicas buscarían generar elecciones de índole académica en las que deberían hacer una labor de rescate y equiparación. Así, por sobre una bibliografía eurocéntrica, podría preferirse una latinoamericana. De la misma forma, en lugar de destacar investigaciones de varones, se podrían destacar las de profesoras. Obviamente sin evitar los referentes recurrentes en ciertos temas, pero una cuestión es conocerlos, estudiarlos y otra muy diferente es hacer de nuestra investigación una mera glosa cargada de falacias de autoridad.

Y no es solo lo que se refiere a las fuentes, los temas también pueden ser tratados del mismo modo afirmativo, optando por los que generan mayor beneficio posible entre los sectores de la población más desposeídos y marginados. Paulo Freire llamó «pedagogía del oprimido» y «pedagogía de la autonomía» a esta labor/movimiento que buscaba revelar la situación de los oprimidos, quienes muchas veces son vistos como objetos, como cosas que no tienen finalidad, o esta es la que los opresores les prescriben. Una metodología crítica para democratizar la investigación científica latinoamericana, a través de una hermenéutica de transformación y emancipación social (Freire, 1993: 157).

Vamos a avanzar un poco más. América Latina puede ofrecer elementos muy interesantes para la investigación contemporánea. Como menciona Silvia Rivera Cusicanqui:

Caminar, conocer, crear: Tanto en aymara como en qhichwa, lo abstracto y lo concreto coexisten estrechamente y a veces son expresados por el mismo término. El nivel de abstracción se relaciona a menudo con un juego de pares opuestos y complementarios [...] un hombre es al mismo tiempo caminante, filósofo, científico, pero además es un agricultor que siembra la comida [...] no solo es un espacio productivo; es también un espacio de construcción activa del orden social-comunal (Rivera, 2015: 208).

Una metodología crítica también redundaría en una cuestión que está a la raíz de toda investigación: la cuestión epistemológica. Nuestras investigaciones son altamente idealistas, se basan y desarrollan modelos que pocas veces repercuten en la realidad, una que además está cargada de relaciones coexistenciales, que es performativa y poderosamente simbólica. 


\section{Conclusión}

Una reflexión metodológica a propósito de la investigación que contribuye a responder de mejor modo las preguntas que surgen en el ámbito del derecho es necesaria para darle un cauce y distribución al trabajo que gira en torno a dicha actividad. De hacerla de cualquier manera a hacerla con causa y conciencia social, al final del día con ética, creo que cualquier observador sensato elegirá la segunda opción. Un asunto ético relevante es que los programas de enseñanza y las lecciones mismas se realizan bajo parámetros de una investigación previa bien elaborada. Los docentes que elaboran malla curricular deberían investigar a fondo acerca de las necesidades sociales y no solo plantear ejercicios, sino también evaluaciones que tengan en cuenta las emociones de los estudiantes, pero también quien imparte clase debería investigar $\mathrm{y}$ adecuar las lecciones a las necesidades del grupo y el contexto.

He tratado de proponer una especie de itinerario que permita constatar que nuestra metodología puede afrontar con responsabilidad social cualquier investigación planteada. Más aún, el planteamiento sería acorde con esta expectativa y lograría generar investigaciones más provechosas, capaces de lograr un resultado simbiótico para superar una ciencia del derecho apática hacia los problemas sociales o, peor aún, una ciencia presuntuosa que jamás se propone un ejercicio crítico porque lo considera desleal.

\section{Referencias}

Deleuze, Gilles y Félix Guattari (1994). Rizoma. Ciudad de México: Coyoacán.

Elgueta, María Francisca y Eric Eduardo Palma (2014). «Una propuesta de clasificación de la clase magistral impartida en la facultad de derecho». Revista chilena de derecho, 41 (3): 907-924. DOI: 10.4067/S0718-34372014000300006.

Estermann, Josef (2014). «Colonialidad, descolonización e interculturalidad. Apuntes desde la filosofía intercultural». Polis. Revista Latinoamericana, 13 (38): 347368. DOI: $10.4067 /$ So718-65682014000200016.

Fernández Jimeno, Natalia (2014). Onfray 5. Cinco aromas dialógicos: lo oculto, lo práctico, lo crítico, la razón de los vencidos y el cuerpo. Universidad de Oviedo.

Freire, Paulo (1993). Pedagogía de la esperanza: Un reencuentro con la pedagogía del oprimido. Madrid: Siglo XXI.

ForD, Donna y John Harris (1992). «The elusive definition of creativity». The Journal of Creative Behavior, 26 (3): 186-196. Disponible en https://bit.ly/2Ekv7Bb.

Grossi, Paolo (1996). Scienza giuridica e legislazione nella sperienza attuale del diritto. Florencia: Quaderni Fiorentini.

Han, Byung Chul (2014). Psicopolítica: Neoliberalismo y nuevas técnicas de poder. Barcelona: Herder. 
Kirchmann, Julius (1983). La jurisprudencia no es ciencia. Madrid: Civitas.

Molina Contreras, Denyz Luz (2009). «Repensar el perfil del abogado en un nuevo modelo de Estado social de derecho y de justicia». Revista mexicana de orientación educativa, 6 (16): 8-15. Disponible en https://bit.ly/38t1NGn.

NúÑEz CUbero, Luis (2006). «Pedagogía emocional: una experiencia de formación en competencias emocionales en el contexto universitario». Cuestiones pedagógicas, 18: 65-8o. Disponible en: https://bit.ly/349afqH.

Nussbaum, Martha (2008). Paisajes del pensamiento: La inteligencia de las emociones. Barcelona: Paidós.

RANCIÈRE, Jean (2008). El maestro ignorante. Buenos Aires: Libros del Zorzal.

RICHARD, Nelly (2000). «Un debate latinoamericano sobre práctica intelectual y discurso crítico». Revista Iberoamericana, 66 (193): 841-850. Disponible en https:// bit.ly/2PdbRvF.

Rivera Cusicanqui, Silvia (2015). Sociología de la imagen. Miradas ch'ixi desde la historia andina. Buenos Aires: Tinta Limón.

Ruiz Resa, Josefa (2017). Innovación docente y nuevas tecnologías. El derecho en la economía del aprendizaje. Valencia. Tirant lo Blanch.

Sivak, Roberto, Adriana Wiater y Fernando Lolas (1997). Alexitimia, la dificultad para verbalizar afectos: Teoría y clínica. Barcelona: Paidós.

Velásquez Valdivia, Anibal (1994). «El proceso creativo: El síndrome de ver lo invisible y realizar lo imposible». Interciencia, 19 (4): 197-204. Disponible en https:// bit.ly/2PcHUfe.

Wolkmer, Antonio Carlos (2017). Teoría crítica del derecho desde América Latina. Madrid: Akal.

\section{Sobre el autor}

José Ramón Narváez Hernández es miembro de la Red de Pedagogía Universitaria y Didáctica del Derecho, así como del comité de la revista del mismo nombre de la Universidad de Chile. Cuenta con el doctorado europeo por investigación en Teoría e Historia del Derecho en la Universidad de Florencia con mención honorífica por la tesis. Además, es licenciado en derecho mención cum laude por investigación científica de la Universidad Panamericana. Ha realizado estancias de investigación en América y Europa. Ha escrito para importantes revistas especializadas en Alemania, Italia, Brasil, Perú, Colombia, Chile, Argentina, España y México. Profesor colaborador de la Universidad de Medellín, Universidad Militar Nueva Granada, Universidad Federal del Paraná y Universidad del Salento, Universidad de Chile, Universidad del Cusco, Universidad Continental de Perú, Universidad Nacional de San Antonio Abad del Cusco, Universidad de Brescia, Italia y Universidad de Granada, España (profesor visitante). Profesor en los posgrados en derecho de las universidades Hidalgo, 
Tlaxcala, Oaxaca, Sinaloa, Michoacán, Chiapas, Cristóbal Colón de Veracruz, UIA de Puebla, en la Escuela Judicial de Campeche y en el programa de diplomados de la Facultad de Estudios Superiores Aragón de la Universidad Nacional Autónoma de México. Doctor honoris causa por la Universidad Andina Nieto Cáceres. Su correo electrónico es iushistoria@gmail.com. (D) https://orcid.org/oooo-0002-0925-3958. 
La Revista Pedagogía Universitaria y Didáctica del Derecho (RPUDD) es una publicación científica semestral que contribuye a la reflexión multidisciplinaria sobre pedagogía universitaria y didáctica del derecho, para la formación y consolidación de esta área de investigación; así como a la difusión de prácticas innovadoras en la enseñanza-aprendizaje del derecho considerando el contexto nacional e internacional. Es una publicación electrónica internacional con una codirección entre Brasil y Chile.

\author{
DIRECTORA \\ María Francisca Elgueta Rosas \\ Universidad de Chile \\ DIRECTOR \\ Renato Duro Dias \\ Universidad Federal de Rio Grande, Brasil \\ SITIO WEB \\ pedagogiaderecho.uchile.cl \\ CORREO ELECTRÓNICO \\ rpedagogia@derecho.uchile.cl \\ LICENCIA DE ESTE ARTÍ́CULO
}

Creative Commons Atribución Compartir Igual 4.0 Internacional

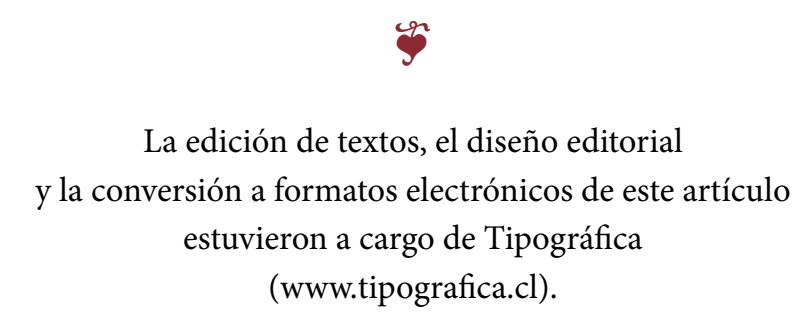

